\title{
Narrativa y formación docente: la experiencia de 5 años de un taller de escritura
}

\section{Narrative and faculty development: results of a five years experience with a creative writing workshop}

Background: Narrative medicine has showed to be a powerful instrument to reinforce relationships, identity, and self-knowledge among health professionals. Subjective issues have been recently recognized as relevant for faculty development in addition to the technical aspects. Since 2006 a creative writing workshop has been included as part of the Diploma in Medical Education at the medical school of the Pontificia Universidad Católica de Chile. Aim: To describe the experience and results of the creative writing workshop (2006-2010). Material and Methods: Descriptive and retrospective study with a qualitative and quantitative design. Thirty-six teachers of the School of Medicine attended a 12-hour workshop. The Kirkpatrick model for evaluation of educational outcomes was used to report the data obtained in the course evaluation survey and in the stories produced. Results: There were positive results at the four levels of Kirkpatrick evaluation model. The learning objectives of the workshop were achieved and 83 stories were created, compiled and published. Conclusions: The creative writing workshop can provide faculty with protected time for reflective practice about academic experiences and produce educational outcomes at different levels of the Kirkpatrick model.

(Rev Med Chile 2012; 140: 659-666).

Key words: Education, professional; Literature; Writing.

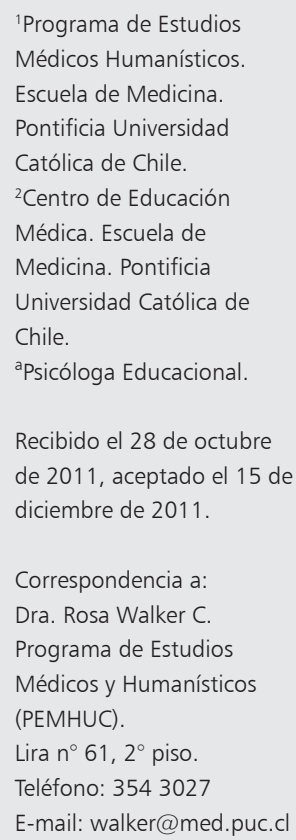

“¿Y qué se aprende escribiendo?

Primero y principal, uno recuerda que está vivo y que eso es un privilegio, no un derecho".

Ray Bradbury, "Zen en el arte de escribir" 1994.

L a formación docente está dedicando últimamente mayor atención a temas como el de pertenencia a la institución, que se han identificado como necesidades relevantes de los docentes $^{1,2}$. El ambiente competitivo y de alta presión por la productividad, y los avances tecnológicos, hacen surgir con mayor fuerza la necesidad de espacios de reflexión para analizar de forma personal los valores y las experiencias de la práctica docente $^{3}$. Se ha buscado en las humanidades, y especialmente en la narrativa, herramientas que faciliten la reflexión ${ }^{4}$.

Las experiencias internacionales de aplicación de la narrativa en medicina de pre y postgrado son numerosas ${ }^{5-9}$. Sin embargo, no existe gran evidencia sobre la sistematización de experiencias en la formación de docentes en Chile; este trabajo podría ser un aporte en nuestro país. 


\section{Medicina narrativa en el contexto de la práctica reflexiva}

La medicina narrativa pertenece a un ámbito más amplio que es la reflexión ${ }^{10,11}$. A continuación, señalamos algunos términos que aparecen con frecuencia relacionados (Figura 1).

\section{Práctica reflexiva ("Reflective practice")}

Schön, autor de uno de los modelos más conocidos de la llamada "práctica reflexiva"12, distingue entre la "reflexión durante la experiencia", de la "reflexión sobre la experiencia". Mann, de una revisión de 29 estudios sobre la práctica de la reflexión, destaca que la reflexión sobre la experiencia permite que "emerjan temas" relevantes para los profesionales, favorece el desarrollo de vínculos significativos que se traducen en la creación de nuevos proyectos, y mejora la capacidad de autosupervisión y autoconocimiento ${ }^{11}$. Sobre este último punto, Hatem y Rider destacan la importancia de comprender cómo las propias emociones y valores afectan la práctica ${ }^{10,13}$. En resumen, la reflexión es fundamental para la educación continua de profesionales, pero se ha reconocido que las metodologías de aplicación están en desarrollo ${ }^{14}$.

\section{Medicina narrativa}

La práctica de la medicina requiere el uso de un lenguaje que vaya más allá de lo empírico: que integre la subjetividad, la afectividad y categorías

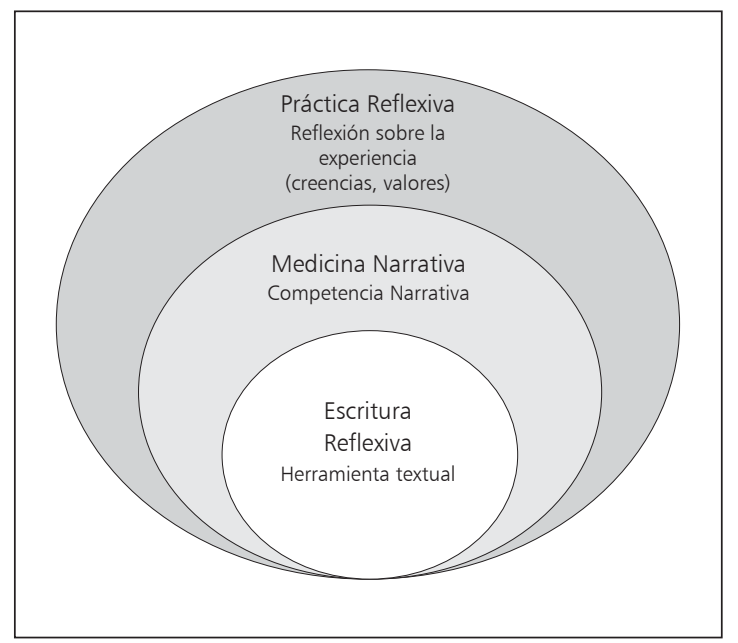

Figura 1. Medicina Narrativa en el contexto de la Práctica Reflexiva.

existenciales como el duelo, el miedo y el sin sentido, que caracterizan el sufrimiento humano ${ }^{15}$. La medicina narrativa es aquella que incorpora un lenguaje narrativo y que se centra en "historias particulares". Estas historias -contadas, leídas o escritas- son "creaciones imaginativas que nacen de la experiencia" 14 . La medicina narrativa tiene su aplicación en la relación médico paciente, en la reflexión sobre sí mismo, en la relación entre colegas (docente-clínica) y con la sociedad ${ }^{16}$. La integración de un lenguaje narrativo se demuestra a través de una "competencia narrativa".

Tabla 1. Ámbitos de aplicación y funciones de la Medicina Narrativa propuestos por Greenhalg T. Y Hurwitz B. (1998)

\begin{tabular}{ll}
\hline En la entrevista médica & - Es la forma natural en que el paciente experimenta y relata la enfermedad \\
diagnóstica & - Promueve la empatía entre el profesional y el paciente \\
& - Permite la construcción de nuevo conocimiento (aporta claves analíticas y categorías útiles) \\
& - Estimula la reflexión \\
\hline En el proceso terapéutico & - Promueve un enfoque integral \\
& - Es por sí mismo intrínsecamente sanador \\
& - Facilita la aparición de otras alternativas de tratamiento \\
En la investigación & - Establece una agenda basada en el paciente \\
& - Desafía el conocimiento tradicional \\
En la educación de & - Facilita la generación de nuevas hipótesis \\
pacientes y profesionales & - Se recuerda fácilmente \\
& - Está basado en la experiencia \\
& - Estimula la reflexión
\end{tabular}




\section{Competencia narrativa}

Rita Charon la define como "un conjunto de habilidades necesarias para reconocer, acoger, interpretar y ser movido por las historias que leemos o escuchamos"16,17. Esta competencia requiere una combinación de habilidades textuales (identificar la estructura de una historia, adoptar múltiples perspectivas, reconocer metáforas y alusiones), habilidades creativas (imaginar diversas interpretaciones, desarrollar la curiosidad e inventar finales alternativos) y afectivas (tolerar la incertidumbre a medida que se desarrolla la historia y entrar en el ánimo de la misma). Estas habilidades, en conjunto, capacitan para entrar en la comprensión del significado de una historia ${ }^{16-18}$.

\section{Escritura reflexiva}

Una forma de desarrollar la competencia narrativa es la escritura reflexiva ${ }^{14}$, técnica escasamente abordada durante la formación médica y que facilita enormemente la toma de concien$\mathrm{cia}^{19}$. Las historias que se van recopilando pueden llegar a constituir un recurso para la docencia y la investigación ${ }^{14}$.

\section{Integración de la narrativa en un programa de formación docente}

Respondiendo a una inquietud del equipo del Diplomado en Educación Médica (DEM) de la Escuela de Medicina de la Pontificia Universidad Católica (EMUC) de aumentar los contenidos humanistas, en 2005 un grupo de docentes inició un seminario de reflexión en torno al libro "Pedagogía del sentido" de Francesc Torralba ${ }^{20}$. Según este autor, ningún acto educativo es neutral, en el sentido que "siempre se comparte una visión de mundo", y se requerirían espacios de reflexión para que los docentes puedan explicitar sus valores, creencias y experiencias: aquello que va constituyendo una comunidad de aprendizaje. Entre los métodos recomendados para la reflexión, Torralba destaca la narrativa. En el 2006 se formalizó el Taller de Narrativa y Docencia como curso optativo del $\mathrm{DEM}^{21}$. El taller ha sido conducido desde entonces por una médico y una psicóloga, ambas con formación en escritura creativa.

\section{Descripción del Taller de Narrativa y Docencia}

El propósito del taller es generar un espacio para la reflexión en el ámbito docente a través de la narrativa. En la Tabla 2 se describen los objetivos y contenidos (Tabla 2).

El taller se estructuró en seis sesiones semanales de dos horas cada una. En estas se abordaron las experiencias de "ser" estudiante y "ser" docente, el "encuentro" docente-alumno y la "experiencia del sufrimiento". Las actividades de cada sesión incluyeron: escritura, lectura y feedback de relatos escritos por los participantes, lectura grupal de textos de literatura universal y reflexión sobre los temas emergentes. Adicionalmente después de cada sesión, las docentes, entregaron por mail comentarios de recapitulación de la sesión, y tareas con ejercicios de lectura y escritura. El material del taller consistió en un manual que contenía el programa, una selección de textos literarios y artículos de medicina narrativa, recursos on-line para escritores, y recomendaciones para la elaboración de textos ${ }^{22}$.

Al finalizar el curso, cada uno de los participantes presentó un relato que fue autoevaluado y evaluado por pares y docentes, utilizando una pauta con 5 indicadores: tema central, presencia de personaje(s), contexto, desarrollo (conflicto y/o transformación) y cumplimiento de normas de redacción, ortografía y estilo.

El objetivo de este artículo es describir la experiencia y los resultados de un Taller de Narrativa y Docencia dirigido a docentes en una Escuela de Medicina chilena.

Tabla 2. Objetivos y contenidos desarrollados en el Taller de Narrativa y Docencia PUC

\begin{tabular}{|ll|}
\hline Objetivos del taller & Contenidos del taller \\
\hline - Experimentar con los textos literarios para despertar la & - Narrativa y medicina \\
creatividad y facilitar la reflexión & - El docente como alumno \\
- Identificar algunos elementos del encuentro docente- & - El docente como tutor y modelo \\
alumno a través de ejercicios narrativos & - Vínculo y sentido del encuentro docente-alumno \\
- Practicar técnicas de escritura creativa & - La experiencia de sufrimiento \\
\hline
\end{tabular}




\section{Metodología}

Estudio descriptivo y retrospectivo que se desarrolló utilizando un diseño cualitativo y cuantitativo.

\section{Participantes}

Un total de 36 docentes de la EMUC asistieron al Taller de Narrativa y Docencia entre 2006 y 2010. Fueron 23 (64\%) hombres, 29 (81\%) médicos y tenían 41,6 \pm 10 años de edad. Con respecto a la categoría académica 10 (43\%) eran Profesores Asistentes.

\section{Evaluación de la experiencia}

Se utilizó el modelo de evaluación de resultados educacionales de Kirkpatrick en cuatro niveles ${ }^{23,24}$. Para el Nivel 1 "Reacción", se utilizó la encuesta semiestructurada de evaluación de la docencia que se aplica al finalizar cada curso del DEM. El instrumento explora la percepción de los asistentes sobre metodología, organización, estilo docente y la apreciación sobre los aspectos más destacados y de mejora del taller. Para el Nivel 2 "Aprendizaje", se utilizaron dos preguntas cerradas de autoevaluación del aprendizaje y una pregunta abierta sobre el interés en cursos de profundización. La confidencialidad se resguardó a través del anonimato de las respuestas y la ausencia de preguntas sobre antecedentes personales.

El Nivel 3 "Conducta", se exploró a través de la producción de relatos. Durante el desarrollo del taller los participantes realizaron una serie de ejercicios de escritura, organizados en torno a cuatro tópicos de docencia: el estudiante, el tutor/ modelo, el encuentro docente-alumno y la experiencia de sufrimiento. El Nivel 4 "Resultados", fue representado por la edición de un libro con una selección de los relatos.

\section{Análisis de la información}

Los resultados de las preguntas cerradas de la encuesta se expresaron en porcentajes.

El análisis cualitativo y descriptivo de los resultados, se realizó de acuerdo a la Teoría Fundadaa ${ }^{25,26}$. A través de la codificación abierta se categorizaron las respuestas abiertas y se identificó el tema central de los relatos. Para asegurar la confiabilidad de los resultados se empleó la estrategia de triangulación de interpretantes ${ }^{27}$.

\section{Resultados}

Respondieron la encuesta de evaluación del taller $32(89 \%)$ de los 36 participantes.

\section{Resultados educacionales}

En relación a la satisfacción con el programa y su organización (Nivel 1 "Reacción" de Kirkpatrick), la mayoría respondió estar de acuerdo con las afirmaciones relacionadas a las características del curso, que las actividades realizadas fueron adecuadas y óptima la calidad de las profesoras (Tabla 3). El clima, los contenidos y la conducción de las docentes fueron los aspectos más valorados. El número de sesiones y actividades fue señalado como insuficiente. Se sugirieron temas de profundización (Tabla 4).

En relación al Nivel 2 "Aprendizaje", el porcentaje promedio de percepción de logro de los objetivos reportados por los encuestados fue $87,3 \%$ y $26(81,3 \%)$ participantes refirieron haber aprendido más de lo esperado.

En relación a la transferencia de lo aprendido a la práctica (Nivel 3 "Conducta"), los participantes generaron 144 relatos, de los cuales 83 (58\%) fueron puestos a disposición del taller voluntariamente por los autores y constituyeron el material de análisis.

Con respecto al efecto del taller a nivel institucional (Nivel 4 de Kirkpatrick), en mayo de 2011 se publicó un libro con 23 relatos, editado por las docentes del taller, que se tituló "Carreras al hospital"28.

\section{Tema central identificado en los relatos}

En la Figura 2, se observa el esquema de clasificación del tema central identificado en cada uno de los 83 relatos.

Los relatos del tópico "El estudiante", se centraron en dos etapas de la vida y sus respectivos desafíos. Por una parte, las historias sobre "la etapa escolar" que tratan de sueños y aventuras, producto de la lectura temprana o de las propias experiencias de juego y relación con otros niños; o la temprana pasión por la biología y el conocimiento, la necesidad de encontrar respuestas a preguntas y explorar. Y por otra parte, las "historias sobre la etapa universitaria de pregrado", que trataron sobre la importancia del sentido de estudiar una carrera de salud y su integración al resto de la vida; la ilusión o desilusión que provocaron 
Narrativa y Formación Docente - M. R. Walker et al

Tabla 3. Resultados de la encuesta de evaluación respondida por los participantes del Taller Narrativa y Docencia de las cohortes 2006-2010. Parte I. Preguntas cerradas*

\begin{tabular}{|c|c|c|c|c|}
\hline Respecto del curso & $\begin{array}{c}\text { Totalmente } \\
\text { de acuerdo } \\
(\%)\end{array}$ & $\begin{array}{c}\text { Parcialmente } \\
\text { de acuerdo } \\
(\%)\end{array}$ & $\begin{array}{c}\text { Totalmente en } \\
\text { desacuerdo } \\
(\%)\end{array}$ & $\begin{array}{l}\text { Omisión } \\
(\%)\end{array}$ \\
\hline Les explicitaron los objetivos a lograr & 91 & 0 & 6 & 3 \\
\hline Los métodos docentes utilizados facilitaron su aprendizaje & 100 & 0 & 0 & 0 \\
\hline El curso trató los temas que esperaba & 69 & 12 & 0 & 19 \\
\hline El curso fue organizado & 88 & 12 & 0 & 0 \\
\hline El curso fue útil para su desempeño docente/profesional & 82 & 18 & 0 & 0 \\
\hline Recomendaría este curso & 94 & 0 & 0 & 6 \\
\hline Realizaría cursos de profundización & 97 & 0 & 3 & 0 \\
\hline Respecto de las actividades realizadas & $\begin{array}{l}\text { Excesivo(a) } \\
(\%)\end{array}$ & $\begin{array}{l}\text { Adecuado(a) } \\
(\%)\end{array}$ & $\begin{array}{l}\text { Insuficiente } \\
\text { (\%) }\end{array}$ & $\begin{array}{l}\text { Omisión } \\
(\%)\end{array}$ \\
\hline En las sesiones, la lectura de textos narrativos le pareció & 0 & 84 & 16 & 0 \\
\hline $\begin{array}{l}\text { En las sesiones, la lectura y apreciación de textos realiza- } \\
\text { dos por los alumnos le pareció }\end{array}$ & 0 & 91 & 9 & 0 \\
\hline En las sesiones, la reflexión sobre la docencia le pareció & 0 & 94 & 0 & 6 \\
\hline Fuera de las sesiones, la lectura de artículos le pareció & 0 & 100 & 0 & 0 \\
\hline Fuera de las sesiones, los ejercicios de escritura le pareció & 0 & 100 & 0 & 0 \\
\hline Respecto de los profesores & $\begin{array}{l}\text { Casi siempre } \\
(\%)\end{array}$ & $\begin{array}{l}\text { A veces } \\
(\%)\end{array}$ & $\begin{array}{c}\text { Casi nunca } \\
(\%)\end{array}$ & $\begin{array}{l}\text { Omisión } \\
(\%)\end{array}$ \\
\hline Su estilo de enseñanza favoreció el aprendizaje & 94 & 6 & 0 & 0 \\
\hline Explicaron con claridad nuevos conceptos & 97 & 3 & 0 & 0 \\
\hline Mantuvieron el interés de los participantes & 100 & 0 & 0 & 0 \\
\hline Realizaron feedback constructivo & 96 & 4 & 0 & 0 \\
\hline Mostraron interés por los intereses del grupo & 100 & 0 & 0 & 0 \\
\hline
\end{tabular}

*Resultados de 32 encuestas que representan el $89 \%$ de los participantes.

personas (amor, enemistad) o situaciones vitales (confusión, errores); o del aprendizaje de valores como el respeto, la fortaleza, la perseverancia, el amor por la naturaleza.

Los temas identificados en los relatos del tópico "El modelo" fueron protagonizados por 3 figuras claves: el profesor, un estudiante o un familiar. El profesor como representante de valores y actitudes personales como la humildad, altruismo, consecuencia, sabiduría y esfuerzo; o como ejemplo de modelo docente por su vocación, pasión para enseñar, disposición y generosidad hacia los alumnos, guía y motor del aprendizaje. El estudiante, como ejemplo de superación y logro ante cualquier circunstancia de la vida. Y un familiar significativo, por las capacidades para enfrentar la vida o representar un ejemplo de apoyo incondicional.
En los relatos del tópico "Encuentro docentealumno" se identifican dos temas relacionados al vínculo: por un lado, como fuente de apoyo que influye en el desarrollo de la identidad personal (confianza y reflexión) o profesional (guía); por otro, como fuente de tensión, por discrepancias en la forma de actuar sea en una situación de aprendizaje (incumplimiento, desmotivación) o en el contexto clínico (toma de decisiones y manejo del error en relación a un paciente o a un par profesional).

Finalmente, en el tópico "La experiencia de sufrimiento" se identificaron temas sobre situaciones dificiles de la propia vida del narrador, ya sea por una relación amorosa o muerte; o de la vida del paciente, ya sea por someterse a largos tratamientos (enfermedad o accidente) o vivir la experiencia del cuerpo como prisión (límite, fragilidad). 
Tabla 4. Resultados de la encuesta de evaluación respondida por los participantes del Taller Narrativa y Docencia de las cohortes 2006-2010. Parte II. Preguntas abiertas*

\begin{tabular}{|c|c|c|}
\hline Preguntas & $\begin{array}{l}\text { Respuestas } \\
\text { n (\%) }\end{array}$ & Categorías de respuesta \\
\hline \multirow{3}{*}{$\begin{array}{l}\text { Lo que más le gustó del Taller de } \\
\text { Narrativa en Docencia } \\
\text { ( } n=50 \text { respuestas de } 30 \text { alumnos) }\end{array}$} & $27(54)$ & $\begin{array}{l}\text { El clima: seguro y participativo, inclusivo, libre y espontáneo. Cita: "El } \\
\text { ambiente grupal para poder compartir cosas a veces más personales" }\end{array}$ \\
\hline & $16(32)$ & $\begin{array}{l}\text { Los contenidos: no corresponden al ámbito médico o están relacio- } \\
\text { nados con la docencia y la narrativa. Cita: "Aprender habilidades de } \\
\text { escritura. Escribir libremente" }\end{array}$ \\
\hline & $7(14)$ & $\begin{array}{l}\text { La conducción de las docentes: entusiasta y motivadora, cálida y } \\
\text { que genera confianza. Cita: "La calidez, acogimiento y cercanía de } \\
\text { las conductoras que generaron un excelente ambiente de grupo" }\end{array}$ \\
\hline \multirow[t]{2}{*}{$\begin{array}{l}\text { Lo que menos le gustó del Taller de } \\
\text { Narrativa en Docencia } \\
\text { ( } n=22 \text { respuestas de } 22 \text { alumnos) }\end{array}$} & $17(77)$ & $\begin{array}{l}\text { Número de sesiones: Insuficientes. Cita: "Insisto. El curso merece } \\
\text { unas horitas más para desenvolver las ricas experiencias que existen } \\
\text { en cada participante" }\end{array}$ \\
\hline & $5(23)$ & $\begin{array}{l}\text { Las actividades (feedback a los trabajos y lectura de relatos): Insu- } \\
\text { ficientes. Cita: "Que casi siempre el rato asignado se hizo poco, no } \\
\text { siempre pudimos leer todos" }\end{array}$ \\
\hline \multirow[t]{3}{*}{$\begin{array}{l}\text { Temas de profundización sugeridos } \\
(\mathrm{n}=25 \text { respuestas de } 25 \text { alumnos })\end{array}$} & $17(68)$ & $\begin{array}{l}\text { Teoría y práctica de las habilidades de escritura: literatura, poesía y } \\
\text { narrativa. Cita: "Profundizar en los distintos estilos narrativos" }\end{array}$ \\
\hline & $5(20)$ & $\begin{array}{l}\text { Aplicación de textos narrativos en docencia. Cita: "Cómo desarrollar } \\
\text { discusiones de libros que se puedan usar para ayudar en docencia" }\end{array}$ \\
\hline & $3(12)$ & $\begin{array}{l}\text { La escritura como espacio de desarrollo personal y reflexión. Cita: } \\
\text { "Escritura, estímulo en la reflexión en el ámbito de la docencia" }\end{array}$ \\
\hline
\end{tabular}

*Resultados de 32 encuestas que representan el $89 \%$ de los participantes.

\section{Discusión}

Los resultados de la experiencia del Taller de Narrativa y Docencia son positivos, y se reflejan tanto en las percepciones que reportaron los participantes como en la producción de relatos.

La percepción de los participantes, tanto en el nivel de "Reacción" como el de "Aprendizaje" tuvieron puntuaciones altas que indican una valoración del método, de las actividades y del estilo docente, así como del cumplimiento de las expectativas de aprendizaje. Sin embargo, los resultados más destacables de esta experiencia tienen relación con las conductas generadas (niveles "Conducta" y "Resultados"). Este cambio se apreció en la transferencia de lo aprendido a la práctica -producción de relatos- $y$ en un logro no esperado del taller, que fue la capacidad de los participantes de "dar el paso" para publicar.

La metodología del taller permitió que los estudiantes cumplieran los objetivos de aprendizaje: experimentar con los textos literarios para despertar la creatividad, identificar algunos elementos del encuentro docente-alumno y practicar técnicas de escritura creativa. Particularmente el segundo objetivo es destacable porque resultó muy generativo para los participantes, la variedad de temas que originó y la profundidad de la reflexión evidencian la huella -como modelo o anti modelo- que es capaz de dejar el tutor, a través de un vínculo profundo o en un encuentro breve en la vida del estudiante.

¿Qué condiciones podrían explicar estos resultados? Tres se identificaron como relevantes: el ambiente seguro, el uso del lenguaje narrativo y los ejercicios de escritura creativa.

En cuanto al ambiente seguro, contar con un espacio donde cada docente pudo encontrarse con "sus preguntas", sentimientos y experiencias fue altamente valorado. Este es un objetivo ligado a la reflexión sobre la experiencia ${ }^{11,12}$ y uno de los principios básicos de la educación de adultos ${ }^{29}$. Crear un ambiente no competitivo es un constante 


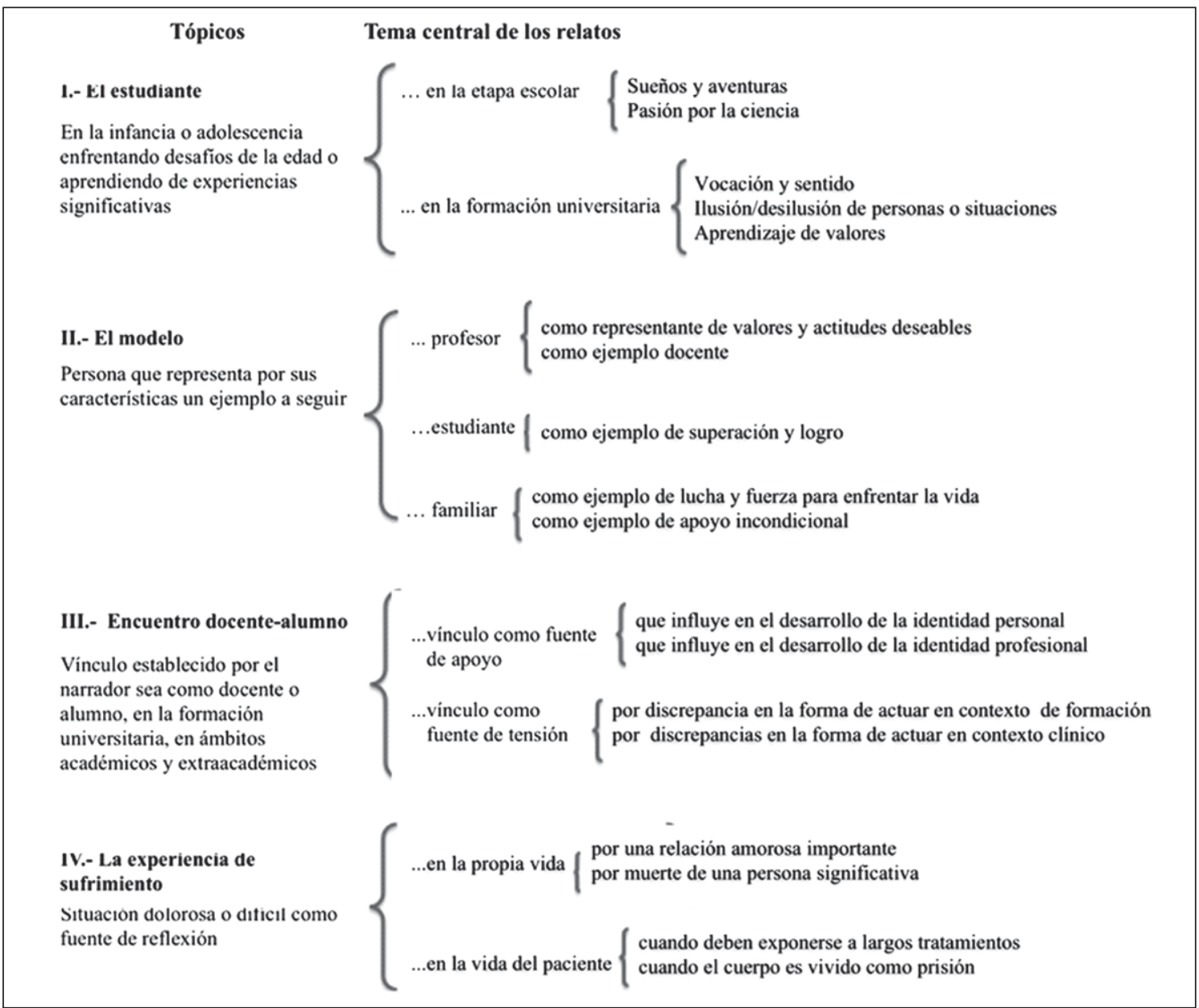

Figura 2. Tema central identificado en 83 relatos escritos por los participantes del Taller de Narrativa y Docencia de las cohortes 2006-2010.

desafío al interior de un taller de escritura creativa; pasar del "quién escribe mejor" a "escuchar la propia voz y reconocer la voz del otro" facilita que salgan a la luz las experiencias. Esta es una de las claves de la "competencia narrativa" que se despliega en la docencia y la práctica médica de calidad ${ }^{12}$.

En relación al uso del lenguaje narrativo, los participantes expresaron en sus relatos aspectos emocionales y subjetivos de las experiencias como docentes o alumnos. Tal como se describe en la literatura, el lenguaje narrativo al permitir explorar el significado subjetivo, contexto y consecuencias de una acción, es un buen facilitador de la reflexión ${ }^{10}$.

Respecto a los ejercicios de escritura realizados, permitieron tomar conciencia de experiencias pasadas y presentes, con un foco personal y auto- biográfico ${ }^{19}$. La "creación de historias basadas en la experiencia", que pone en juego la creatividad, es una actividad central en la práctica reflexiva ${ }^{14}$.

El clima, lenguaje y ejercicios contribuyen a que el taller de narrativa constituya una oportunidad para un "procesamiento de las experiencias" como búsqueda del sentido dentro de una comunidad de aprendizaje ${ }^{11}$.

En cuanto a limitaciones metodológicas, el análisis de los resultados se basó en 36 participantes; sin embargo, los hallazgos reflejan la percepción de la mayoría del universo considerado. En la metodología cualitativa utilizada, sólo se realizó el primer nivel de codificación de los tres propuestos por la Teoría fundada, considerando que el estudio es sólo descriptivo. 
En conclusión, un taller de narrativa para docentes proporciona un espacio protegido para la práctica reflexiva en torno a las experiencias académicas y produce resultados educacionales. La divulgación de los resultados de un nuevo taller de formación docente puede ser un aporte a la educación médica chilena. En el futuro, sería importante generar un banco de relatos y estimular a los autores a publicar sus obras, medir el impacto de la intervención en la práctica docente y explorar nuevos espacios (música, arte, etc).

Agradecimientos. Al Dr. Vicente Valdivieso por su extraordinaria inspiración. A todos los participantes que han confiado en nosotras y de quienes hemos aprendido tanto.

\section{Referencias}

1. Pololi LH, Dennis K, Winn GM, Mitchell JA. Needs assessment of medical school faculty: caring for the caretakers. J Contin Educ Health Prof 2003; 23: 21-9.

2. Higgs J, MCallister L. Educating clinical educators: using a model of the experience of being a clinical educator. Med Teach 2007; 29: e51-7.

3. Scannell K. Writing for our lives. Ann Intern Med 2002; 137: 779-81.

4. Pololi LH, Frankel R. Humanising medical education through faculty development: linking self-awareness and teaching skills. Med Educ 2005; 39: 154-62.

5. Montgomery K, Charon R, Coulehan J. The study of literature in medical education. Acad Med 1995; 70: 787-94.

6. Shapiro J, Duke A, Boker J, Ahearn CS. Just a spoonful of humanities makes medicine go down: introducing literature into a family medicine clerkship. Med Educ 2005; 39: 605-12.

7. Charon R. What to do with stories. The science of narrative medicine. Can Fam physician 2007; 53: 1265-7.

8. Greenhalgh T, Hurwitz B, editors. Narrative based medicine. Dialogue and discourse in clinical practice. London: BMJ Books; 1998.

9. Kidd MG, Connor JTH. Striving to do good things: Teaching humanities in canadian medical schools. J Med Humanit 2008; 29: 45-54.

10. Hatem D, Rider E. Sharing stories: narrative medicine in an evidence-based world. Patient Educ Couns 2004; 54 : 251-3.

11. Mann K, Gordon J, MacLeod A. Reflection and reflective practice in health professions education: a systematic review. Adv in Health Sci Educ 2009; 14: 595-621.

12. Schon D. The reflective practitioner. San Francisco: Jossey-Bass; 1983.

13. Hatem D, Ferrara E. Becoming a doctor: fostering humane caregivers through creative writing. Patient Educ Couns 2001; 45: 13-22.

14. Bolton G. Reflective practice. Writing and professional development. London: SAGE Publications; 2001.

15. Walker R. Desarrollo de la capacidad empática a través de las humanidades. Ars Médica 2003; 2. Disponible en: http://escuela.med.puc.cl/publ/ArsMedica/ArsMedica2/14_Walker.html [Consultado el 22 de octubre de 2011].

16. Charon R. Narrative medicine. A model for empathy, reflection, profession and trust. JAMA 2001; 286: 18971902.

17. Charon R. What Narrative Competence is for. AJOB 2001; $1: 1$.

18. Charon R. Narrative and Medicine. NEJM 2004; 350: 862.

19. Carretero M, Bennett N, Jarvinen A, Pope M, Ropo E. Procesos de enseñanza y aprendizaje. Buenos Aires: Aique; 1998.

20. Torralba F. Pedagogía del sentido. Madrid: Ediciones PPC; 1997.

21. Triviño X, Sirhan M, Moore P, Montero L. Impacto de un programa de formación en docencia en una escuela de medicina. Rev Med Chile 2011; 139: 1527-34.

22. Hagel J. Saber y contar. Producción de textos narrativos. Santiago: Ediciones UC; 1999.

23. Steinert Y, Mann K, Centeno A, Dolmans D, Spencer J, Gelula $\mathrm{M}$ et al. A systematic review of faculty development initiatives designed to improve teaching effectiveness in medical education: BEME Guide No. 8. Med Teach 2006; 28: 497-526.

24. Triviño X, Sirhan M, Moore P, Reyes C. Formación en educación de los docentes clínicos de medicina. Rev Med Chile 2009; 137: 1516-22.

25. Flick U. Introducción a la investigación cualitativa. Madrid: Morata; 2004.

26. Strauss A, Corbin J. Basics of qualitative research. Grounded theory procedures and techniques. Newbury Park: Sage; 1990.

27. Patton M. Qualitative evaluation and research methods. Newbury Park: Sage; 1990.

28. Walker R, Zúñiga D, editoras. Carreras al hospital. Vol. I (2006-2010). Santiago: Ediciones Lulu.com; 2011.

29. Vella J. Learning to listen-learning to teach: the power of dialogue in educating adults. San Francisco CA: JosseyBass; 2002. 